\title{
A simultaneous minimally invasive approach to treat a patient with coronary artery disease and metastatic lung cancer
}

\author{
Yuanhao $\mathrm{Fu}^{1}$, Lufeng Zhang ${ }^{1}$, Ling $\mathrm{Ji}^{2}$, Chenyang $\mathrm{Xu}^{2}$ \\ ${ }^{1}$ Third Hospital of Peking University, Beijing, China \\ 2People's Hospital of Ganzhou, Nanchang University, Ganzhou, China
}

Videosurgery Miniinv 2016; 11 (4): 300-303

DOI: https://doi.org/10.5114/wiitm.2016.63987

\begin{abstract}
Concurrent lung cancer and coronary artery disease requiring treatment with percutaneous coronary intervention or coronary artery bypass grafting is not rare. An individualized perioperative anticoagulation regimen and minimal surgical trauma will benefit the patient's postoperative recovery. We successfully treated a 68-year-old female patient with a lesion in the left anterior descending artery and metastatic right lung carcinoma by simultaneous minimally invasive direct coronary artery bypass grafting via a small left thoracotomy and thoracoscopic wedge resection of the lung lesion. She recovered and was discharged on the eighth postoperative day. The patient showed no symptoms of myocardial ischemia postoperatively. Computed tomography scan did not indicate metastatic lesion of lung carcinoma at 1-year follow-up. In conclusion, minimally invasive direct coronary artery bypass grafting combined with thoracoscopic wedge resection is an effective minimally invasive treatment for concurrent lung cancer and coronary artery disease. This technique eliminates the risk of perioperative bleeding and provides satisfactory mid-term follow-up results.
\end{abstract}

Key words: simultaneous management, minimally invasive surgery, video-assisted thoracoscopic surgery, coronary artery bypass graft surgery.

\section{Introduction}

Concurrent lung cancer and coronary artery disease requiring treatment with percutaneous coronary intervention $(\mathrm{PCl})$ or coronary artery bypass grafting (CABG) is not rare in clinical practice [1]. An individualized perioperative anticoagulation regimen and minimal surgical trauma will benefit the patient's postoperative recovery. Dual antiplatelet therapy after $\mathrm{PCl}$ may induce intraoperative and postoperative bleeding. Conventional CABG via sternotomy may add operative trauma in elderly and weak patients. We herein report a minimally invasive approach involving a combination of minimally invasive direct coronary artery bypass (MIDCAB) and thoracoscopic wedge resection of a right lung lesion for simultaneous treatment of coronary artery disease and lung cancer.

\section{Case report}

A 68-year-old woman with a history of colon cancer was referred for thoracoscopic resection of a metastatic lesion in the right inferior pulmonary lobe (Photo 1). Preoperative coronary angiography revealed total occlusion of the left anterior descending artery (LAD) after the origin of the first diagonal branch (Photo 2). Percutaneous coronary intervention was not performed because of its technical difficulty and contraindication for perioperative anti- 

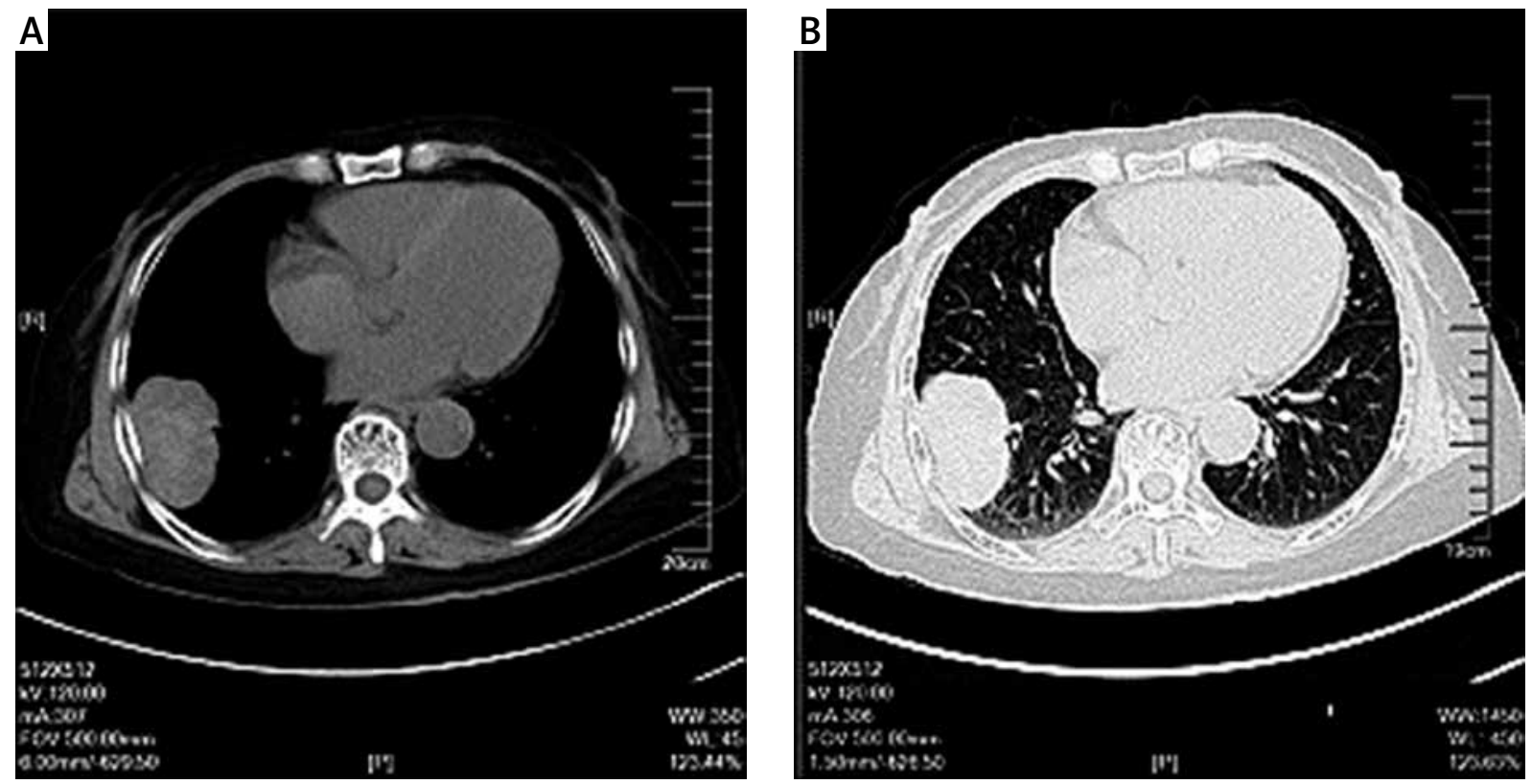

Photo 1. Chest computed tomography revealed a metastatic lesion in the right inferior pulmonary lobe
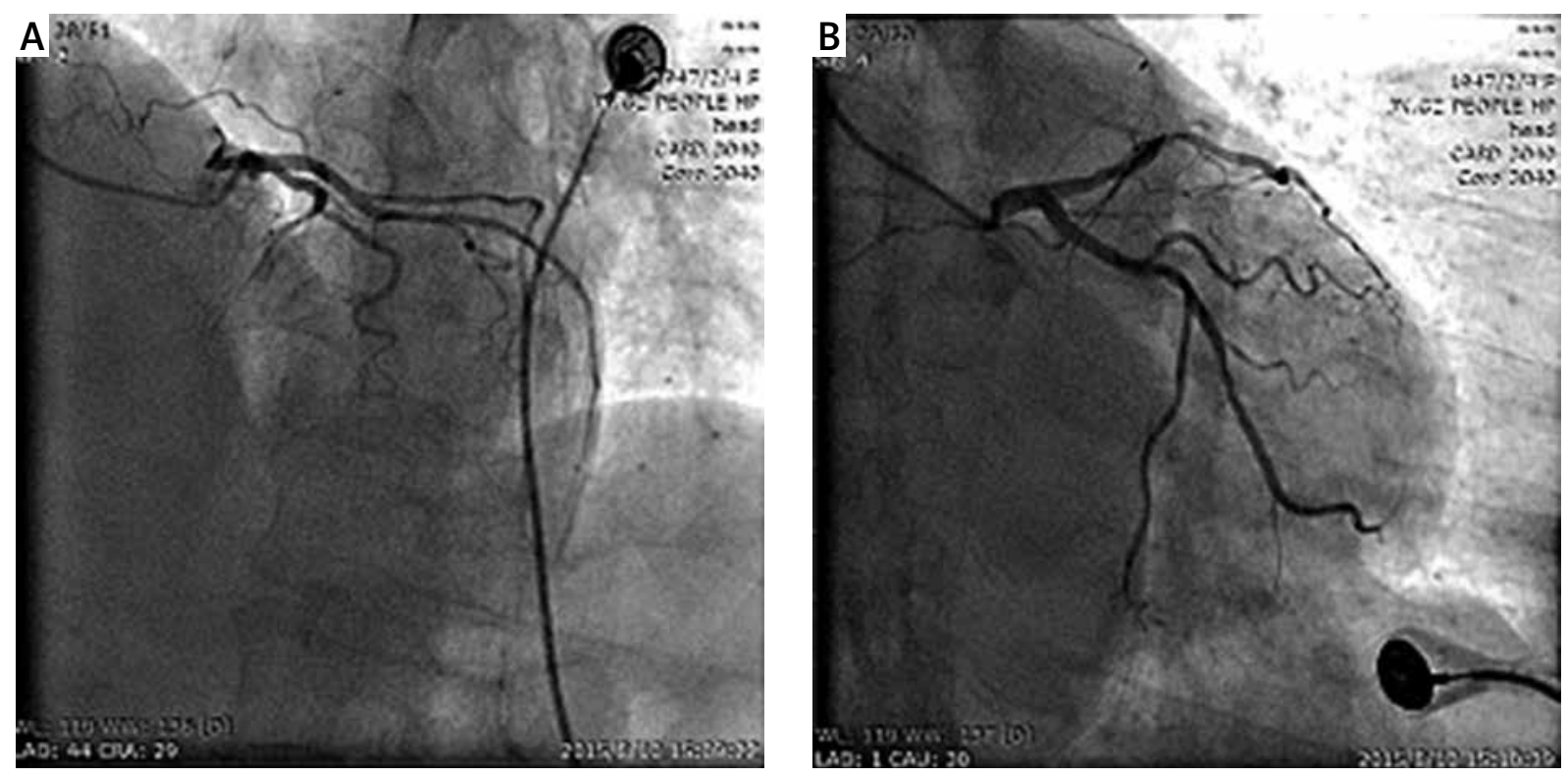

Photo 2. Coronary angiography revealed total occlusion of the left anterior descending artery after the origin of the first diagonal branch

coagulation. To minimize the operative trauma, we performed simultaneous MIDCAB via a small left thoracotomy and thoracoscopic wedge resection of the lung lesion.

The patient was anesthetized and intubated with a double-lumen endotracheal tube. Single-lung ventilation was used to facilitate exposure for harvesting of the left internal thoracic artery. The patient was positioned supine with $30^{\circ}$ rotation toward the right decubitus position by means of a rolled towel. External pads for emergency defibrillation were placed on the right anterior and left posterior sides of the chest wall. A 5-cm left anterior thoracotomy incision was then created along the inframammary line and tunneled into the fourth intercostal space according to the preoperative chest $\mathrm{X}$-ray and coro- 
nary angiography images. After harvesting of the left internal thoracic artery, a pericardial incision was made to expose and stabilize the LAD with a specially designed minimally invasive stabilizer. The coronary anastomosis was performed using a continuous running suture technique (8-0 suture) and intracoronary shunting.

After heparin neutralization and switching of the single-lung ventilation to the other side, the patient was positioned supine with $30^{\circ}$ rotation toward the left decubitus position. A 4-cm incision in the fifth intercostal space was made along the right anterior axillary line for wedge resection of the dorsal segment of the right inferior pulmonary lobe using a thoracoscopic linear stapler with a blue reload. After the surgery, the patient was transferred to the intensive care unit and recovered well. She was discharged on postoperative day 8. A perioperative red blood cell transfusion was not necessary. The patient's postoperative mechanical ventilation time was 12 h. Postoperative transthoracic echocardiography showed a normal ejection fraction and heart chamber. Pathologic examination of the lung lesion showed a moderately differentiated metastatic colonic adenocarcinoma. The lesion was positive for cytokeratin (CK), CK20, Ki-67, CDX2, and carcinoembryonic antigen as determined by immunohistochemistry.

The patient was clinically well at the 1-year follow-up visit. She was in NYHA I without symptoms of myocardial ischemia. Chest computed tomography showed no indication of a lung mass at the 1-year follow-up.

\section{Discussion}

Concomitant coronary artery disease and lung cancer is not uncommon and may pose a serious surgical dilemma [1, 2]. No formal consensus regarding the optimal treatment of patients with cancer and coronary artery disease, whether by contemporary or staged surgical treatment, has been reached $[3,4]$. Cancer resection followed by CABG may increase the perioperative and intraoperative cardiogenic risk to the patient, while CABG followed by cancer resection is associated with a risk of intraoperative carcinoma metastasis. Combined onestage cancer resection and CABG may be associated with a high risk of surgical trauma to the patient. A one-stage, minimally invasive combined surgical procedure provides a new option for these patients.
The advantages of a one-stage approach include use of fewer anesthetic agents, less operative stress and pain, a shorter hospital stay, lower therapeutic cost, and less delay of tumor treatment [2-5]. Minimally invasive direct coronary artery bypass for revascularization of the LAD has become a routine operation in clinical practice. Minimally invasive direct coronary artery bypass is associated with substantially lower risks of cancer metastasis, intraoperative hemorrhage, sudden inflammatory response syndrome, and pulmonary edema than is cardiopulmonary bypass [6-8]. Furthermore, MIDCAB without sternotomy can provide similar long-term graft patency and satisfactory outcomes $[6,8]$. In the present case, the combination of MIDCAB and thoracoscopic wedge resection of the metastatic lung lesion had excellent short- and mid-term results.

\section{Conclusions}

Minimally invasive direct coronary artery bypass combined with thoracoscopic wedge resection of the pulmonary lobe is an effective minimally invasive treatment for concurrent lung cancer and coronary artery disease. This technique eliminates the risk of perioperative bleeding and provides satisfactory mid-term follow-up results.

\section{Conflict of interest}

The authors declare no conflict of interest.

\section{References}

1. Rao V, Todd TR, Weisel RD, et al. Results of combined pulmonary resection and cardiac operation. Ann Thorac Surg 1996; 62: 342-7.

2. Dyszkiewicz W, Jemielity M, Piwkowski C, et al. The early and late results of combined off-pump coronary artery bypass grafting and pulmonary resection in patients with concomitant lung cancer and unstable coronary heart disease. Eur J Cardiothorac Surg 2008; 34: 531-5.

3. Saxena P, Tam RK. Combined off-pump coronary artery bypass surgery and pulmonary resection. Ann Thorac Surg 2004; 78: 498-501.

4. Dyszkiewicz W, Jemielity MM, Piwkowski CT, et al. Simultaneous lung resection for cancer and myocardial revascularization without cardiopulmonary bypass (off-pump coronary artery bypass grafting). Ann Thorac Surg 2004; 77: 1023-7.

5. Elami A, Korach A, Rudis E. Lung cancer resection or aortic graft replacement with simultaneous myocardial revascularization without cardiopulmonary bypass. Chest 2001; 119: 1941-3.

6. Holzhey DM, Jacobs S, Mochalski M, et al. Seven-year follow-up after minimally invasive direct coronary artery bypass: expe- 
rience with more than 1300 patients. Ann Thorac Surg 2007; 83: 108-14.

7. Jaffery Z, Kowalski M, Weaver WD, et al. A meta-analysis of randomized control trials comparing minimally invasive direct coronary bypass grafting versus percutaneous coronary intervention for stenosis of the proximal left anterior descending artery. Eur J Cardiothorac Surg 2007; 31: 691-7.

8. Zhang L, Cui Z, Song Z, et al. Minimally invasive direct coronary artery bypass for left anterior descending artery revascularization - analysis of 300 cases. Videosurgery Miniinv 2015; 10: 548-54.

Received: 30.06.2016, accepted: 15.11.2016. 